\title{
Application of personalized medicine to chronic disease: a feasibility assessment
}

\author{
Ruslan Dorfman ${ }^{1,2^{*}}$, Zayna Khayat ${ }^{2,3}$, Tammy Sieminowski ${ }^{7}$, Brian Golden ${ }^{3}$ and Renee Lyons $s^{4,5,6}$
}

\begin{abstract}
Personalized Medicine has the potential to improve health outcomes and reduce the cost of care; however its adoption has been slow in Canada. Bridgepoint Health is a complex continuous care provider striving to reduce the burden of polypharmacy in chronic patients. The main goal of the study was to explore the feasibility of utilizing personalized medicine in the treatment of chronic complex patients as a preliminary institutional health technology assessment. We analyzed stroke treatment optimization as a clinical indication that could serve as a "proof of concept" for the widespread implementation of pharmacogenetics. The objectives of the study were three-fold:

1. Review current practice in medication administration for stroke treatment at Bridgepoint Health

2. Critically analyze evidence that pharmacogenetic testing could (or could not) enhance drug selection and treatment efficacy for stroke patients;

3. Assess the cost-benefit potential of a pharmacogenetic intervention for stroke.

We conducted a review of stroke treatment practices at Bridgepoint Health, scanned the literature for drug-gene and drug-outcome interactions, and evaluated the potential consequences of pharmacogenetic testing using the ACCE model.

There is a substantial body of evidence suggesting that pharmacogenetic stratification of stroke treatment can improve patient outcomes in the long-term, and provide substantial efficiencies for the healthcare system in the short-term. Specifically, pharmacogenetic stratification of antiplatelet and anticoagulant therapies for stroke patients may have a major impact on the risk of disease recurrence, and thus should be explored further for clinical application. Bridgepoint Health, and other healthcare institutions taking this path, should consider launching pilot projects to assess the practical impact of pharmacogenetics to optimize treatment for chronic continuous care.
\end{abstract}

Keywords: Stroke; Stroke treatment; TIA; Pharmacogenetics; Statins; Clopidogrel; Warfarin; Antihypertensive; Antidepressants; Patient outcomes

\section{Introduction}

Personalized medicine tailors medical treatment to a patient's personal history, genetic profile and specific biomarkers [1,2]. Pharmacogenetics (PGx) is an application of personalized medicine, referring to the process where patients are stratified and treated based on their genetic profile, which is used to assess expected drug response and the risk of adverse side effects. Traditional medicine typically relies on the broad application of "standard of

\footnotetext{
* Correspondence: ruslan.dorfman@geneyouin.ca

${ }^{1}$ Present Address: Geneyouin Inc, Maple, ON, Canada

${ }^{2}$ The Rotman School of Management, The University of Toronto, Toronto, ON, Canada

Full list of author information is available at the end of the article
}

care" or "one size fits all" treatments to all patients with a given diagnosis, irrespective of their genetic context. The association between one's gene variants and drug response rates or risks of adverse side effects to specific medications have been reported in many studies; in response, the US Food and Drug Administration (FDA) has updated the labels of nearly 100 drugs with recommendations for genetic testing prior to their use [3].

A personalized medicine approach to drug treatment decision-making offers several potential benefits. The approach may reduce the rate of adverse drug reactions (ADR) [4], which would likely improve treatment adherence. Greater treatment adherence may then prevent 
disease recurrence or the onset of secondary complications, leading to better clinical and economic outcomes [5]. Additional economic benefits would be gained by limiting the prescription and reimbursement of costly treatments to only those patients who are most likely to respond positively [6]. Despite these potential benefits, the challenge has been translating the many recent discoveries of PGx markers associated with a specific drug response into routine medical practice. In Canada, progress towards including PGx in clinical practice has lagged many peer OECD countries due to a fragmented regulatory environment and lack of reimbursement models [7]. For example the approval and reimbursement of PGx tests is decided separately in each province, leading to long delays for adoption of the technology. In order for strong regulatory guidelines to be created, it is important to consider the feasibility of PGx in clinical settings.

The Bridgepoint Collaboratory for Research and Innovation, the research institute of Bridgepoint Health (BPH), was established to improve the hospital's understanding of chronic complex diseases (CCD), develop approaches to improving care and integrate advanced research into practice. To investigate the feasibility of using PGx in a clinical setting, we evaluated the potential implications of PGx on stroke treatment at BPH. BPH is what is known as a complex continuing care facility. The majority of patients at BPH are afflicted with multiple chronic illnesses and are often on multiple medications, posing the additional problem of polypharmacy and its associated potential increase in ADRs. Stroke is the third most common condition treated at BPH. The condition has a significant treatment burden due to the need for prolonged rehabilitation and long-term need for multiple medications. Thus the potential impact of introducing the precision of PGx on improving patient outcomes and healthcare costs for this population is significant and worthy of rigorous exploration.

In Canada, more than 50,000 strokes occur every year, making stroke the leading cause of long-term disability. The financial cost of treatment, including direct treatment and patient/caregiver productivity costs, is estimated to average $\$ 50,000$ per person within the first six months alone. Average costs per patient range from $>\$ 17 \mathrm{~K}$ for mild transient ischemic attack (TIA) episodes, to $>\$ 53 \mathrm{~K}$ for ischaemic and $>\$ 56 \mathrm{~K}$ for haemorrhagic stroke [8]. The annual economic burden of stroke in Canada is estimated to be $\$ 2.5$ billion, or $\sim 1 \%$ of the country's GDP [9]. All stroke patients require continuous medication following discharge in order to mitigate their constant risk of stroke recurrence [10,11]. An individual who has suffered a stroke has a $20 \%$ chance of having another stroke within two years, and $50 \%$ of patients have a second episode within 5 years of the initial stroke. Substantial costs for medication are borne not only by an institution (like BPH) during a patient's hospital stay but, particularly in the long-term, by patients and payers who must finance medications and other therapies that are prescribed upon discharge. Therefore, even a small reduction in stroke recurrence risk or severity has the potential to yield significant economic benefits.

Although the potential value proposition of applying PGx at BPH could is significant, to date no trial has been conducted to survey the clinical validity of PGx stratification in a complex continuing care context. Complex care patients are typically excluded from randomized clinical trials due to their multiple co-morbidities. However, complex care patients are ideal candidates for PGx stratification [12] which can not only improve quality and cost of care in the hospital, but can also benefit outpatients in perpetuity. A major known risk factor for stroke recurrence is inadequate dosing of anticoagulant medication in patients with atrial fibrillation. With adequate anticoagulation therapy at least $9 \%$ of strokes could be prevented [13], however the Canadian Stroke Registry Network found that only $10 \%$ of atrial fibrillation stroke sufferers were medicated and had a coagulation index within the advised therapeutic range [14]. Additionally, medications used for stroke prevention are also used for patients with other symptoms and diagnoses at BPH - PGx optimization of stroke medication can therefore be of potential value to multiple patient populations.

For this study, we assessed the potential clinical and economic impact of PGx on preventing stroke recurrence. We conducted semi-structured interviews of healthcare providers, reviewed the literature on PGx genes and stroke, and modeled health and economic outcomes. Our assessment confirmed the initial hypothesis that PGx can be used to optimize medication use by chronically ill patients, minimize the risk of patients' health deterioration, and reduce the associated in-patient and out-patient costs to the Canadian healthcare system, and the broader economy. We suggest that complex continuing care institutions consider performing proof of concept trials to investigate the use of PGx. These studies will help demonstrate the value of PGx for clinical implementation and may inform clinical guideline recommendations.

\section{Methods}

To assess the impact of personalized medicine we addressed three main areas: drugs, genes and consequences. We sought to answer the following questions:

1. Drugs: What is the current standard of care drug treatment for stroke patients? We conducted semi-structured interviews with hospital's physicians and pharmacists to determine drug treatments used 
for stroke patients and clinical protocols used for selecting treatment and dosing regimens for prevention of secondary stroke.

2. Genes: Which genes are linked to responses to current drug treatments for stroke?

We reviewed the literature to identify potential genetic associations with current stroke treatment responses. We queried the PubMed database with the following key words: "stroke risk factors", "stroke treatment", "stroke AND pharmacogenetics", "stroke AND gene", "warfarin AND gene", "clopidogrel AND gene", "statin AND gene", "stroke AND cost effectiveness". Peer-reviewed publications spanning 2005 - Feb 2012 were reviewed.

3. Consequences: What are the potential health and economic outcomes of applying PGx to current stroke treatment protocols? The Office of Public Health Genomics of the Centers for Disease Control in the U.S. has developed the ACCE Model $[15,16]$ for evaluation of clinical utility, analytic utility, and clinical validity of genetic diagnostic tests for various diseases [17]. We applied this comprehensive framework to critically evaluate PGx tests used for the selection and dosing of treatments for stroke patients undergoing rehabilitation. The pharmacogenetic tests that were evaluated were screened for genes identified in (2) above. Notably, in this overview we do not provide technical details on specific risk alleles for each gene; only replicated and validated pharmacogenetic markers were considered. Cost efficiency and sensitivity analyses were coded and modeled in Excel. Cost efficiency analyses included data from the published literature and cost of drugs according to the Ontario Drug Benefit Plan formulary. The prices for new medications that have not been added to the Ontario formulary were extrapolated from price of the drug in US market. The sensitivity analysis explored factors determining the cost-efficiency of pharmacogenetic stratification.

\section{Research findings \\ Four main classes of pharmacological treatments are prescribed at BPH for secondary prophylaxis in stroke patients}

Current clinical practices for stroke treatment at $\mathrm{BPH}$ reflect current Canadian Best Practice Recommendations for Stroke Care [18]. These interventions mitigate the key risk factors such as atrial fibrillation, diabetes, hypertension, and hyperlipidemia. Stroke patients receive medications from the following four classes for continuous stroke prevention [19-25]:
1. Antiplatelet control: aspirin or clopidogrel.

2. Continuous anticoagulant treatment: primarily warfarin for patients with atrial fibrillation (10-15\% of all stroke patients), or heparins for patients with deep vein thrombosis prophylaxis (administered to the majority of stroke patients during hospitalization).

3. Hypertension control: angiotensin-converting enzyme (ACE) [26] inhibitors or angiotensin receptor blockers are administered to the majority of patients due to additional benefits of these medications.

4. Statins: prescribed to the majority of stroke patients primarily as anti-inflammatory agents and for the control of hyperlipidemia, [27-30].

Key pharmacogenetic genes implicated in the efficacy of drugs used to treat stroke

Each of the four classes of drugs used for stroke treatment were analyzed in depth to evaluate the potential impact of PGx on their optimal use:

\section{Antiplatelet analysis}

Antiplatelet medications, which block platelet activation and reduce blood clots are used in virtually all patients at risk of cardiovascular disease. The vast majority of $\mathrm{BPH}$ stroke patients are on a single antiplatelet medication, predominantly acetylsalicylic acid (ASA). Table 1 summarizes potential pharmacogenetic genes, i.e., genes variants that may affect how a patient responds to antiplatelet treatments.

\section{Aspirin}

Approximately $50 \%$ of patients at $\mathrm{BPH}$ are treated with Aspirin [31] at varying doses (80-325 mg). ASA is regarded as ineffective for those patients who still suffer a stroke while medicated with ASA. As an alternative, those patients are typically prescribed the antiplatelet agent clopidogrel for thrombosis prevention.

\section{Clopidogrel}

Clopidogrel $\left(\right.$ Plavix $\left.^{\circ}\right)$ is a pro-drug that requires activation by the cytochrome P450 CYP2C19 enzyme. Depending on ethnicity, up to $25 \%$ of patients have mutations in the CYP2C19 gene that result in a less active or inactive 2C19 enzyme, potentially leading to reduced drug response. In March 2010, the FDA issued a "Black Box" warning for clopidogrel, citing that about $14 \%$ of patients are poor metabolizers of the drug due to CYP2C19 gene variants. Patients with decreased drug efficacy have a 1.5-3 times greater risk of stroke or death compared to patients who metabolize the drug. This lead the FDA to recommend genetic testing to identify patients who are poor metabolizers [32]. Additional genetic variants which affect clopidogrel metabolism have recently been identified. 
Table 1 Antiplatelet medications and potential pharmacogenetic genes

\begin{tabular}{|c|c|c|c|c|}
\hline Drug name & Brand name & Potential pharmacogenetic genes & PubMed ID & Details \\
\hline \multirow[t]{4}{*}{ Acetylsalicylic acid } & \multirow[t]{4}{*}{ Aspirin } & \multirow[t]{4}{*}{ NTRK1; LTC4S; ITGB3 } & 21390260; & \multirow[t]{4}{*}{ Risk of ADR and lower response-associated alleles } \\
\hline & & & 19862937; & \\
\hline & & & 12816731; & \\
\hline & & & 19390185 & \\
\hline \multirow[t]{11}{*}{ Clopidogrel } & \multirow[t]{11}{*}{ Plavix } & \multirow[t]{11}{*}{ CYP2C19; PON1; P2RY12; ABCB1; } & 22153531; & \multirow{11}{*}{$\begin{array}{l}\text { Risk or phenotype-associated alleles. Any combination } \\
\text { of two of CYP2C19 loss-of-function alleles is associated } \\
\text { with diminished platelet response and worse } \\
\text { cardiovascular outcomes }\end{array}$} \\
\hline & & & 22088980; & \\
\hline & & & 22028352; & \\
\hline & & & 21881565; & \\
\hline & & & 21716271; & \\
\hline & & & 21497813; & \\
\hline & & & 21332306; & \\
\hline & & & 21170047; & \\
\hline & & & 20801498; & \\
\hline & & & 20126830; & \\
\hline & & & 19637098 & \\
\hline Prasugrel* & Effient & \multirow[t]{2}{*}{ None } & & Better suited for patients who cannot metabolize clopidogrel \\
\hline Dipyridamoles-ASA & Aggrenox & & & \\
\hline
\end{tabular}

"Prasugrel is not on the Ontario or British Columbia public formularies, therefore economics were analyzed based on US pricing.

Variations in the $A B C B 1$ gene may impact the drug's efficacy by affecting its absorption [33]. Common variants within the PON1 gene (Q192R) affect the drug's activation with QQ192 homozygous individuals exhibiting a higher risk of stent thrombosis than RR192 homozygous individuals [34]. However this finding was not replicated and thought to be a result of interaction with cigarette smoking [35]. Comprehensive pharmacogenetic tests based on pharmacogenetic genes can identify poor metabolizers who are at increased risk of stroke when taking clopidogrel and would therefore require alternative therapies.

\section{Prasugrel}

The alternative antiplatelet drug prasugrel (Effient ${ }^{\oplus}$ ), approved in Canada in 2010, is much more expensive than generic clopidogrel and was found to be associated with a higher risk of mortality from bleeding. However, the drug is not affected by genetic variations in the CYP2C19 gene, and produces a consistent response in patient populations. It is unclear whether clopidogrel or prasugrel is more effective in preventing stroke recurrence and heart attacks. Recent studies indicate that prasugrel is more effective than clopidogrel, however these studies did not account for poor clopidogrel metabolizers in the patient population [36-41]. Pharmacogenetic tests would ensure that patients receive the treatments they are most likely to respond to and could limit the unnecessary use of prasugrel, thereby sparing patients the associated bleeding risk unless the drug is deemed necessary (ie, for non-clopidogrel responsers). Using pharmacogenetics to tailor treatment decision for patients who must chose between these two drug options would reduce the risk of recurrent stroke through improved anticoagulation control. The international Clinical Consortium for Pharmacogenetic Implementation has released recommendations advising pharmacogenetic stratification when considering these drugs [42].

\section{Cost-effectiveness of applying PGx to clopidogrel}

The cumulative average health system cost of ischemic stroke treatment for the first six months in Ontario exceeds $\$ 53 \mathrm{~K}$ [8] while the cost distribution is substantially higher for severe stroke patients. The cost for stroke patients with a Modified Rankin Score of 4, approaches \$100 K and doubles for patients with a Score of 5. Stroke recurrence, when preventative treatment fails due to the patient being a poor metabolizer of the prescribed drug or inadequate dosing of antiplatelet medications, has a major impact on the healthcare system. A recent cost-benefit analysis compared CYP2C19 genotype-guided antiplatelet therapy to treatment with clopidogrel or prasugrel regardless of patient genotype [43]. The results indicated that genotypeguided antiplatelet therapy was more effective and less costly compared to treating all patients, regardless of genotype, with clopidogrel (ICER -\$6760 [95\% (CI) -\$6720 to $-\$ 6790]$ ) or prasugrel (ICER - $\$ 11,710$ [95\% confidence interval (CI) $-\$ 11,480$ to $-\$ 11,950]$ ] [43]. A similar cost/ benefit profile from applying pharmacogenetics to clopidogrel/prasugrel treatment was demonstrated in a study 
of patients with acute coronary syndrome undergoing planned percutaneous coronary intervention [44].

Our cost-effective and sensitivity analyses were aimed at identifying the major factors that determine the efficiency of PGx stratification (Table 2). The model is based on the current distribution of antiplatelet medications being prescribed to $\mathrm{BPH}$ patients: $50 \%$ of stroke patients are currently treated with ASA, 10\% are put on Dipyridamole/ ASA (Aggrenox ${ }^{\circ}$ ) and the remaining $40 \%$ receive clopidogrel. We compared three alternative scenarios: current treatment protocol vs pharmacogenetic stratification vs random. We assumed that only $80 \%$ of the clopidogrel users metabolize the drug and the $20 \%$ non-metabolizers are at increased risk of stroke recurrence due to ineffective anticoagulation treatment. We also assumed that application of a genetic test would identify the majority of these non-metabolizers, who would then be prescribed prasugrel. Switching clopidogrel non-responders to prasugrel would reduce the overall stroke recurrence risk for the entire stroke population from $8.2 \%$ to $7.4 \%$. The cost of PGx genetic testing of $\$ 600$ per patient in the clopidogrel subgroup (averaged cost $\$ 250$ ) would be offset by avoiding the additional treatment cost of a secondary stroke.
To assess the factors affecting the robustness and sensitivity of this model we modified several variables. The direct cost of PGx testing is not a significant factor and nullified the positive effect of clopidogrel stratification only if the cost exceeded $\$ 5,100$. Surprisingly the PGx test sensitivity, i.e. the ability to correctly identify poor clopidogrel metabolizers turned out to be the most sensitive parameter in the model. The PGx approach ceases to be cost effective when more than $21 \%$ of patients continue on clopidogrel despite being poor metabolizers. Therefore, healthcare providers and policy makers must consider PGx test sensitivity in their patient population in order to achieve the maximum clinical and economic benefits of pharmacogenetic stratification, even at higher costs of genetic testing.

\section{Summary of pharmacogenetics applied to antiplatelet medications}

Conducting a pharmacogenetic-based patient stratification prior to prescribing clopidogrel has the potential to improve treatment efficacy and yield substantial long-term economic benefits for stroke patients and health care funders. As of 2012, clopidogrel has been generalized, resulting in lower costs compared to prasugrel and

Table 2 Cost comparisons of current standard of care for stroke patients at BPH compared with pharmacogenetic stratification (PGx) or a randomized alternative

\begin{tabular}{|c|c|c|c|c|}
\hline $\begin{array}{l}\text { Proportion of stroke patients treated } \\
\text { with particular medication }\end{array}$ & Average annual cost $\$$ & Base case (standard of care) & PGx stratified & Alternative random \\
\hline ASA & 27 & 0.5 & 0.5 & 0.5 \\
\hline Plavix & 941 & 0.4 & 0.32 & 0.2 \\
\hline Aggrenox & 601 & 0.1 & 0.1 & 0.1 \\
\hline Effient (estimated cost) & 1110 & 0 & 0.08 & 0.2 \\
\hline Proportion of untreated & & 0.08 & 0.016 & 0.04 \\
\hline Recurrence risk untreated & & 0.2 & 0.2 & 0.2 \\
\hline Recurrence risk treated & & 0.072 & 0.072 & 0.072 \\
\hline Cost of treatment failure & & 53,576 & 53,576 & 53,576 \\
\hline Probability of failure & & 0.082 & 0.074 & 0.077 \\
\hline Risk adjusted failure cost ${ }^{*}$ & & 4406 & 3967 & 4132 \\
\hline Incremental annual cost of treatment & & 450 & 463 & 484 \\
\hline Cost of PGx test & & 0 & 250 & 0 \\
\hline Total costs Year 1 & & 4856 & 4680 & 4615 \\
\hline Discounted costs Year $2^{* *}$ & & 4714 & 4301 & 4481 \\
\hline Discounted costs Year $3^{* *}$ & & 4577 & 4176 & 4350 \\
\hline Discounted costs Year $4^{* *}$ & & 4444 & 4055 & 4224 \\
\hline Discounted costs Year $5^{* *}$ & & 4314 & 3936 & 4101 \\
\hline Cumulative costs: & & 22905 & 21149 & 21771 \\
\hline Differential savings & & 0 & 1756 & 1134 \\
\hline
\end{tabular}

*Average cost of ischemic stroke treatment in Ontario according to Goeree et al, 2005.

Please note that the costs are substantially higher for more severe stroke patients: costs for patients with Modified Rankin Score $4=100,000 \$ C D N$ and score $5=200,000 \$ C D N$.

${ }^{* *} \mathrm{NPV}$ calculation assumes $3 \%$ annual interest rate. 
subsequent savings for the healthcare system on a per pill basis. If clopidogrel dosage is adjusted according to patients' metabolic profiles, this may result in further savings (not modeled as part of this study).

\section{Anticoagulation analysis}

Warfarin is used broadly in patients with atrial fibrillation, heart valve replacement, recent heart attack, and for venous thromboembolism prophylaxis in patients who have undergone hip or knee replacements. However, warfarin has a narrow therapeutic range: at low doses it does not decrease the risk of stroke, while at higher doses it significantly increases the risk of intracranial bleeding $[22,45]$. Proper warfarin dosing is challenging due to variation in VKORC1 and CYP2C9 genes (Table 3), which impact the clinical efficacy of the medication [46-49].

A pharmacogenetic approach for accurate warfarin dosing has been developed based on known variations in VKORC1 and CYP2C9 genes [50]. PGx testing can explain $\sim 50 \%$ of the dose variance in Caucasians and can reduce the time necessary to determine optimal INR (International Normalized Ratio of prothrombin time) in the first few days of drug initiation. Although this reduces incidence of out-of-range INRs, there is little benefit for PGx testing beyond the first two weeks of treatment initiation for patients with empirically established dosage.

Some pharmacogenetic tests are less effective in Asians and African Americans due to inability to capture additional pharmacogenetic variants in these populations. The use of ethnically-optimized biomarkers can further improve PGx-based dose prediction algorithms in these populations [51], allowing continuous and safe utilization effective warfarin treatment. Clinical Pharmacogenetics Implementation Consortium's guidelines for warfarin dosing have been published supporting the application of genetic testing prior to drug initiation [52].

Prospective clinical trials have investigated the use of a pharmacogenetic approach for warfarin dosage in cohorts of orthopedic patients [53] and patients with atrial fibrillation [54]. The studies demonstrated the superiority of the PGx approach over the current standard of care in reducing the number of adverse effects through genetically guided warfarin dosing within the first days of hospitalization. Additional pharmacogenetic trials of warfarin dosing are currently underway (NCT00927862).

\section{Alternative anticoagulant therapeutics}

Dabigatran etexilate (PRADAX $\left.{ }^{\mathrm{m}}\right)$, which was recently approved by Health Canada, has similar efficacy to warfarin but is considered a safer alternative [55], as the drug is not currently known to have pharmacogenetic interactions. However, dabigatran was associated with an increased incidence of gastrointestinal adverse reactions, (35\% vs. $24 \%$ for warfarin) and no competitive inhibitors for dabigatran are available.

\section{Cost-effectiveness of warfarin and dabigatran}

Dabigatran is ten times more expensive than warfarin [56,57], although the higher cost could be offset by a reduction in secondary stroke risks and associated treatment cost, and absence of INR monitoring, which is required for warfarin users. However, as these cost evaluations did not compare dabigatran against warfarin pharmacogenetic protocols [58] it is still unclear whether dabigatran is indeed more cost-effective than warfarin [56].

An extensive cost-effectiveness analysis conducted by Lutter et al. [59] estimated that genetic testing for warfarin therapy for an individual patient can reduce

Table 3 Anticoagulant treatments and genes with variants that potentially affect drug response

\begin{tabular}{|c|c|c|c|c|}
\hline Drug name & Brand name & Pharmacogenetic genes & PubMed ID reference & Details \\
\hline \multirow[t]{10}{*}{ Warfarin } & \multirow[t]{10}{*}{ Coumadin } & \multirow{10}{*}{$\begin{array}{l}\text { CYP2C9; VKORC1; CALU; } \\
\text { CACNA1C; CYP4F2 }\end{array}$} & 20200517 & \multirow{10}{*}{$\begin{array}{l}\text { Risk or phenotype-associated alleles. Weekly warfarin } \\
\text { dose requirements were lower in those with CYP2C9 } \\
\text { loss-of-function alleles as compared with the wild type } \\
\text { CYP2C9 genotype }\end{array}$} \\
\hline & & & 19794411; & \\
\hline & & & 20072124 & \\
\hline & & & 17341693; & \\
\hline & & & 18250228 & \\
\hline & & & 20203262 & \\
\hline & & & 18535201; & \\
\hline & & & 19297519; & \\
\hline & & & 19300499; & \\
\hline & & & 18535201 & \\
\hline Dabigatran* & \multirow[t]{2}{*}{ Pradax } & & & \\
\hline Heparin & & & & \\
\hline Enoxaparin & Lovenox & & & \\
\hline
\end{tabular}


healthcare costs by $\$ 550$ ( $\$ 900$ savings minus $\$ 350$ for the cost of genetic testing). For the U.S. healthcare system, net savings from warfarin PGx could be about $\$ 1.1$ billion annually while even with BPH's smaller patient population scale, the long-term savings to the healthcare system could be substantial [24].

\section{Summary: pharmacogenetics applied to anticoagulants}

Although PGx-guided selection of warfarin dosage could be more cost-effective than alternative treatment with dabigatran, the impact of warfarin pharmacogenetics is difficult to assess due to highly variable effects caused by non-genetic factors such as diet. However, if stroke patients undergo genetic testing for clopidogrel-metabolizing genes, the additional cost of genotyping VKORC1 and CYP2C9 genes would be very low.

\section{Antihypertensives analysis}

The clinical efficacy of the remaining classes of stroke treatment medications such as angiotensin-converting enzyme inhibitors, angiotensin II receptor (AGTR2) antagonists, and statins are also affected by genetic variation in metabolic genes, as well as in genes encoding the drug's main targets and proteins involved in the signaling cascades (Table 3). Most stroke patients are prescribed ACE inhibitors for blood pressure control and the anti-inflammatory effects that reduce the risk of stroke recurrence $[21,60]$. ACE inhibitors have known ADRs, including hypokalemia and drug-induced dry cough and can be substituted with AGTR2 antagonists. Occasionally, administration of ACE or AGTR2 blockers even at the maximal therapeutic doses is insufficient to control hypertension. In such cases, additional medication is prescribed alongside a high dose of ACE or angiotensin II receptor blockers. Simultaneous administration of several antihypertensive compounds the polypharmacy effect, thus contributing to the cumulative risk of ADRs.

\section{Pharmacogenetics of antihypertensive medications}

Although different ACE inhibitors, as well as AGTR2 antagonists, have different pharmacogenetic profiles (Table 4), physicians and especially pharmacists generally do not differentiate between antihypertensive medications within the same therapeutic class -i.e. an ACE inhibitor could be switched for a ACE blocker by a pharmacist, depending on availability, without considering potential differences in pharmacogenetic profiles, as these medications are considered to be therapeutically interchangeable. Pharmacogenetic profiling for antihypertensive drugs is not very cost-effective because treatment optimization through adjustment of drug and/or dose and blood pressure monitoring is easy and inexpensive.

\section{Analysis of statins}

Some statins, especially simvastatin, are known to cause severe muscle or bone pain, and in some patients can result in severe muscle waiting and kidney failure. Statins exhibit differential pharmacogenetic profiles, and the risk of muscle damage can be alleviated by switching to a different brand. Like antihypertensive drugs, the efficacy of statins can be monitored by testing blood cholesterol and adverse side effects are frequently eliminated by substitution to another brand with a different pharmacogenetic profile. Pharmacogenetic genes implicated in patients' responses to statins include $S L C O 1 B 1$ and $A B C C 1$. Both SLCO1B1 and ABCC1 transporters mediate drug uptake from the gastrointestinal tract and genetic variants alter drug uptake, resulting in differing drug responses. For instance, SLCO1B1 variants block the uptake of flavonoids present in grapefruit juice and statins and missense variants were shown to be associated with simvastatin-induced myopathy $[61,62]$. Pharmacogenetic testing for $S L C O 1 B 1$ variants can significantly reduce the incidence of simvastatin-induced myopathy and allow for wider and safer use of the drug [63], which is the cheapest among statins. However, use of PGx for the selection of statin treatment is not expected to have a major impact on the efficiency of stroke prevention. Therefore, this test could be a useful add-on to other genes in the genetic testing panel, but should not be pursued as a stand-alone test.

\section{Summary: pharmacogenetics of antihypertensives and statins}

PGx may reduce the rate of undesired side effects of antihypertensive drugs and statins, resulting in better treatment adherence after discharge. However, the clinical efficacy of statins, ACE and AGTR2 blockers is more markedly affected by changes in cholesterol levels and blood pressure, respectively. In this case, PGx has a limited impact on drug selection and stroke recurrence risk and recovery. PGx testing for these drugs is typically included in a more extensive screening panel, as the cost of including these variants in a genetic test is negligible.

\section{Added value of expanded pharmacogenetic testing}

At $\mathrm{BPH}$, antidepressants are frequently prescribed to help both stroke and general practice patients cope with debilitating chronic diseases. Like clopidogrel and warfarin, antidepressants are metabolized by the CYP family of proteins and response to some antidepressants, as well as risk of adverse side effects is linked to CYP2C19 and CYP2D6 metabolic activity. Citalopram, the most commonly used antidepressant at $\mathrm{BPH}$, is primarily metabolized by both the cytochrome enzymes, and patients who have gain-offunction mutations in these genes are fast metabolizers and exhibit a poor response to citalopram. Genetic testing for variants in the CYP2D6 and CYP2C19 genes can be 
Table 4 Antihypertensives and statins: genes with variants that potentially affect the drug response rate or the risk of adverse side effects

\begin{tabular}{|c|c|c|c|c|}
\hline Drug name & Brand name & Pharmacogenetic genes & PubMed ID & Effect \\
\hline \multicolumn{5}{|c|}{ ACE inhibitors } \\
\hline Captopril & Capoten & AGTR1 & 18347611 & $\begin{array}{l}\text { Specific variants (s.v.) associated } \\
\text { with improved outcomes }\end{array}$ \\
\hline Enalapril & Vasotec; Renitec & & & \\
\hline Ramipril & Altace; Tritace; & & & \\
\hline Perindopril & Coversyl; Aceon & AGTR1; BDKRB1 & 20712529; 20712529 & $\begin{array}{l}\text { S.v. associated with improved } \\
\text { outcomes }\end{array}$ \\
\hline Lisinopril & Listril; Lopril; Novatec; & AGTR1; CLCN6; NPPA & $18347611 ; 18212314$ & $\begin{array}{l}\text { S.v. associated with improved } \\
\text { outcomes }\end{array}$ \\
\hline \multicolumn{5}{|c|}{ AGTR2 antagonists } \\
\hline Losartan & $\begin{array}{l}\text { Cozaar; Hyzaar; Lacidipine; } \\
\text { Lortaan }\end{array}$ & CYP2C9 & $11823761 ; 11408373$ & $\begin{array}{l}\text { CYP2C9*3 allele is associated } \\
\text { with a reduced rate of drug } \\
\text { metabolism }\end{array}$ \\
\hline Irbesartan & Avalide; Avapro; Irbesarran; & APOB; LDLR & $15453913 ; 15453913$ & $\begin{array}{l}\text { S.v. associated with improved } \\
\text { outcomes }\end{array}$ \\
\hline Candesartan & Amias; Atacand; Blopress; & KNG1 & 19584173 & $\begin{array}{l}\text { S.v. associated with improved } \\
\text { outcomes }\end{array}$ \\
\hline Valsartan & Diovan & & & \\
\hline Telmisartan & Micardis; Pritor & & & \\
\hline \multicolumn{5}{|l|}{ Statins } \\
\hline Atorvastatin & Lipitor; Torvast & $\begin{array}{l}\text { CYP3A4; ABCB1; HTR7;HTR3B; } \\
\text { GNB3; USP5; SLCO1B1; ABCC1 }\end{array}$ & $\begin{array}{l}\text { 17600820; 18851956; 19833260; } \\
18851956\end{array}$ & $\begin{array}{l}\text { Risk or phenotype-associated } \\
\text { alleles }\end{array}$ \\
\hline Rosuvastatin & Crestor & CYP2C8; CYP2C19 & 20178046 & \\
\hline Simvastatin & Zocor; Lipex & $\begin{array}{l}\text { CYP3A4; HCR; SLCO1B1; AGTR1; KIF6; } \\
\text { HTR7; HTR3B; GNB3; USP5; ITGB3; }\end{array}$ & $\begin{array}{l}\text { 11250978; 20403997; 18073581; } \\
\text { 18551043; 18222353; 18222354; } \\
18222355 ; 17600820 ; 11545752\end{array}$ & $\begin{array}{l}\text { Risk or phenotype-associated } \\
\text { alleles }\end{array}$ \\
\hline
\end{tabular}

accomplished using existing CYP panels. A broad CYP450 genotyping or ADME screening panel could potentially improve selection of antidepressant treatments.

\section{Summary of results}

The PGx-guided treatment approach has the potential to optimize drug treatment for complex continuing care patients who take a battery of medications for a prolonged period of time. We have shown that genetic testing for antiplatelet medications and anticoagulants would greatly benefit prevention of secondary stroke, particularly in patients on clopidogrel or warfarin. Specifically, PGx testing for clopidogrel metabolism is strongly recommended by the FDA and leading pharmacogenetic experts. It would be prudent of $\mathrm{BPH}$ to consider practical implementation of genetic testing for current and future clopidogrel users. Furthermore, an integrated approach for critical re-evaluation of the entire drug portfolio can add further value at minimal extra cost when expanding basic testing to include other gene variants.

\section{Discussion}

A pharmacogenetic treatment stratification could be critical to improving treatment efficacy and cost-efficiency by optimizing drug selection and dosing according to a patient's genetic profile. This method is an evidence-based approach and relies on scientifically developed correlations between responses to medications and specific genetic variants. Once the methodology for treatment optimization is established for a particular disease, the same model can be applied for the evaluation and reassessment of other chronic complex diseases.

Polypharmacy is a major issue in CCD treatment, as patients typically receive multiple medications concurrently. In Canada, 1.3 million seniors take five or more medications, and in complex care $80 \%$ of patients are concurrently treated with three or more medications [64]. High pill burden leads to poor treatment adherence and contributes to an increased risk of ADRs. Additionally, polypharmacy is a known morbidity and mortality risk factor. Up to $12 \%$ of seniors taking multiple medications visit the hospital for an ADR each year. The direct medical costs of ADRs exceed $\$ 100$ billion annually in the U.S. alone. Implementation of pharmacogenetics in CCD treatment may help to reduce the polypharmacy burden $[65,66]$, and provide specific recommendations to physicians that may help to reduce the error rate in drug prescriptions. 
The current study shows that PGx can improve selection and dosing for at least two of the four classes of drugs commonly prescribed to stroke patients at $\mathrm{BPH}$, particularly clopidogrel and warfarin, since these medications can substantially reduce the risk of stroke recurrence. PGx testing for ACE inhibitors and statins is less critical to risk of stroke recurrence, although it could reduce the burden of polypharmacy. PGx-driven treatment optimization is feasible and might have substantial value for multiple conditions as many medications are being used for separate diseases that share the same comorbidities. However, in many instances there is still ongoing debate about the strength of evidence of PGx indications and no clear cost-effectiveness analysis that includes simultaneous modeling of multiple medications and comorbidies which are relevant for complex care. It is unlikely that clinical trials for PGx-driven complex care will be carried out in the near future. Nevertheless, in order to improve care for its patients, BPH needs to consider practical implementation of pharmacogenetics in routine clinical practice in a step-wise manner.

To further investigate the value of PGx testing, a pilot study could be performed using extended genetic testing with the ADME panel. This would help determine the initial dosage of warfarin and allow better use of antihypertensive and antidepressant medications. The economic benefits of such an approach are expected to be substantial and long-lasting. Additionally, long-term research studies with patient follow-up are needed to ensure patient safety in PGx trials and coordination of care. The accompanying economic analysis for PGx-driven treatment optimization has to take into consideration all medications that may differ from patient to patient, necessitating the development of more comprehensive and flexible models.

In theory, PGx could be used in a multitude of clinical settings and disease conditions, however significant barriers exist to broader implementation including: 1) lack of clear clinical guidelines, 2) cost and reimbursement for genetic tests, 3) rapidly evolving technology that needs to overcome regulatory barriers, 4) the need for integration with electronic patient records and better predictive algorithms and 5) training of physicians in genetics, pharmacogenetics and genetic counselling. From our informal discussions with BDP's physicians it was clear that the clinical team lacked sufficient pharmacogenetics knowledge and the right tools for supporting decision-making. Also, there is no clear reimbursement system to pay for genetic testing.

The direct costs of genetic testing are only a small fraction of the costs associated with personalized medicine in complex care. While proof of concept trials for PGxdriven optimization could be funded through research grants, under the current hospital funding system all costs for personalized medicine will have to come from the hospital's operations budget. From a purely economic perspective for $\mathrm{BPH}$ and other healthcare institutions, there is no financial incentive to support the implementation of PGx approaches despite the huge potential for improving patient outcomes and the long-term costs of care through reduction of polypharmacy and risk of ADRs. In order for PGx approaches to be successfully implemented, future funding guidelines need to take into account the long-term benefits of treatment improvement programs.

\section{Take away messages}

- PGx can improve patient outcomes by reducing the risk of stroke recurrence and severe side effects

- PGx has the potential to directly reduce costs of drug treatments both during patient hospitalization and post-discharge

- PGx can have an immediate and long-term impact on patient outcomes and could substantially reduce both direct and indirect healthcare costs while improving quality of life

- PGx implementation requires a long-term outlook on patient outcomes and necessitates revision of hospital funding initiatives

- Extensive CYP or ADME screening panels that test for a large number of medications provide added value and could be more cost-effective to current standard of care

\section{Competing interests}

Renee Lyons, Tammy Sieminowski and Brian Golden - none; Ruslan Dorfman, is a founder and CEO of Geneyouin which is a direct to consumer provider of genetic testing and consulting services; in the past two years Zayna Khayat was employed as a management consultant at SECOR and KPMG LLP for public and private Canadian healthcare system and received fees over $\$ 10,000$.

\section{Authors' contributions}

$\mathrm{RD}$ and ZK conducted literature review, interviews and drafted the manuscript; TS conducted literature overview and assisted in manuscript writing; RL and BG supervised the project, provided strategic direction and assistant in manuscript writing. All authors read and approved the final manuscript.

\section{Acknowledgements}

Ruslan Dorfman held a CIHR "Science to Business" Scholarship. We would like to thank Alexis Schaink, research analyst, and Janice Takata-Shewchuk, Head of Pharmacy, of Bridgepoint Health for data collection and analyses.

\section{Author details}

${ }^{1}$ Present Address: Geneyouin Inc, Maple, ON, Canada. ${ }^{2}$ The Rotman School of Management, The University of Toronto, Toronto, ON, Canada. ${ }^{3}$ International Centre for Health Innovation, Richard Ivey School of Business, University of Western Ontario, London, ON, Canada. ${ }^{4}$ Bridgepoint Chair in Complex Chronic Disease Research, TD Scientific Director, Bridgepoint Collaboratory for Research and Innovation, Toronto, Ontario, Canada. ${ }^{5}$ Dalla Lana School of Public Health, The University of Toronto, Toronto, ON, Canada. Institute of Health Policy,

Management and Evaluation, University of Toronto, Toronto, Ontario, Canada.

${ }^{7}$ Neurorehabilitation, Bridgepoint Hospital, Toronto, Ontario, Canada.

Received: 2 May 2013 Accepted: 25 September 2013

Published: 18 December 2013

\section{References}

1. Dancey JE, Bedard PL, Onetto N, Hudson TJ: The genetic basis for cancer treatment decisions. Cell 2012, 148:409-420. 
2. Evans WE, Relling MV: Moving towards individualized medicine with pharmacogenomics. Nature 2004, 429:464-468.

3. Table of pharmacogenomic biomarkers in drug labels. http://www.fda.gov/ drugs/scienceresearch/researchareas/pharmacogenetics/ucm083378.htm.

4. Vidal F, Gutierrez F, Gutierrez M, Olona M, Sanchez V, Mateo G, Peraire J, Vilades C, Veloso S, Lopez-Dupla M, Domingo P: Pharmacogenetics of adverse effects due to antiretroviral drugs. AIDS Rev 2010, 12:15-30.

5. Malhotra AK, Zhang JP, Lencz T: Pharmacogenetics in psychiatry: translating research into clinical practice. Mol Psychiatry 2011, 17:760-769.

6. Yan L, Beckman R: Pharmacogenetics and pharmacogenomics in oncology therapeutic antibody development. Biotechniques 2005, 39:S565-S568.

7. Meckley LM, Neumann PJ: Personalized medicine: factors influencing reimbursement. Health Policy 2010, 94:91-100.

8. Goeree R, Blackhouse G, Petrovic R, Salama S: Cost of stroke in Canada: a 1-year prospective study. J Med Econ 2005, 8:147-167.

9. Strokes cost Canada .5B a year: study. http://www.cbc.ca/news/health/story/ 2010/06/07/stroke-cost.html.

10. Yip S, Benavente O: Antiplatelet agents for stroke prevention. Neurotherapeutics 2011, 8:475-487.

11. Simmons BB, Yeo A, Fung K: Current guidelines on antiplatelet agents for secondary prevention of noncardiogenic stroke: an evidence-based review. Postgrad Med 2010, 122:49-53.

12. Frueh FW: Back to the future: why randomized controlled trials cannot be the answer to pharmacogenomics and personalized medicine. Pharmacogenomics 2009, 10:1077-1081.

13. Evans A, Davis S, Kilpatrick C, Gerraty R, Campbell D, Greenberg P: The morbidity related to atrial fibrillation at a tertiary centre in one year: $9.0 \%$ of all strokes are potentially preventable. J Clin Neurosci 2002, 9:268-272.

14. Gladstone DJ, Bui E, Fang J, Laupacis A, Lindsay MP, Tu JV, Silver FL, Kapral MK Potentially preventable strokes in high-risk patients with atrial fibrillation who are not adequately anticoagulated. Stroke 2009, 40:235-240.

15. Kroese M, Zimmern RL, Farndon P, Stewart F, Whittaker J: How can genetic tests be evaluated for clinical use? experience of the UK genetic testing network. Eur J Hum Genet 2007, 15:917-921.

16. Zimmern RL, Kroese M: The evaluation of genetic tests. J Public Health (Oxf) 2007, 29:246-250

17. ACCE model system for collecting, analyzing and disseminating information on genetic tests. http://www.cdc.gov/genomics/gtesting/ACCE/FBR/index.htm.

18. Coutts S, Kelloway L: Stroke prevention. In CANADIAN BEST PRACTICE RECOMMENDATIONS FOR STROKE CARE. Edited by Lindsay MP, Gubitz G, Bayley M, Phillips S; 2012

19. Aw D, Sharma JC: Antiplatelets in secondary stroke prevention: should clopidogrel be the first choice? Postgrad Med J 2012, 88:34-37.

20. Shulga O, Bornstein N: Antiplatelets in secondary stroke prevention. Front Neurol 2011, 2:36.

21. Gustavsen GP: ACE inhibitors prevent stroke in high-risk patients, independent of blood pressure-lowering effect. J Fam Pract 2002, 51:595.

22. Hylek EM, Skates SJ, Sheehan MA, Singer DE: An analysis of the lowest effective intensity of prophylactic anticoagulation for patients with nonrheumatic atrial fibrillation. N Engl J Med 1996, 335:540-546.

23. McCabe DJ, Brown MM: Prevention of ischaemic stroke-antiplatelets. Br Med Bull 2000, 56:510-525.

24. Rose AJ, Berlowitz DR, Ash AS, Ozonoff A, Hylek EM, Goldhaber-Fiebert JD: The business case for quality improvement: oral anticoagulation for atrial fibrillation. Circ Cardiovasc Qual Outcomes 2011, 4:416-424.

25. Selim M: Antiplatelets for stroke prevention: implications of the PRoFESS trial. Stroke 2009, 40:1936-1937.

26. Wallace RW: Pharmacogenomics: the next logical step. Drug Discov Today 1999, 4:105-107.

27. East JE: Statin therapy after stroke or transient ischemic attack. N Engl J Med 2006, 355:2368-2369. author reply 2370-2361.

28. Elkind MS: Statin therapy after stroke or transient ischemic attack. N Engl J Med 2006, 355:2369. author reply 2370-2361.

29. Kent DM: Stroke-an equal opportunity for the initiation of statin therapy. N Engl J Med 2006, 355:613-615.

30. Hebert PR, Gaziano JM, Chan KS, Hennekens CH: Cholesterol lowering with statin drugs, risk of stroke, and total mortality. An overview of randomized trials. JAMA 1997, 278:313-321.

31. Arai T, Sekizawa K, Ohrui T, Fujiwara H, Yoshimi N, Matsuoka H, Sasaki H: ACE inhibitors and protection against pneumonia in elderly patients with stroke. Neurology 2005, 64:573-574.
32. FDA drug safety communication: reduced effectiveness of plavix (clopidogrel) in patients who are poor metabolizers of the drug. http:/www.fda.gov/drugs/ drugsafety/PostmarketDrugSafetyInformationforPatientsandProviders/ ucm203888.htm

33. Mega JL, Close SL, Wiviott SD, Shen L, Walker JR, Simon T, Antman EM, Braunwald E, Sabatine MS: Genetic variants in ABCB1 and CYP2C19 and cardiovascular outcomes after treatment with clopidogrel and prasugrel in the TRITON-TIMI 38 trial: a pharmacogenetic analysis. Lancet 2010, 376:1312-1319.

34. Bouman HJ, Schomig E, van Werkum JW, Velder J, Hackeng CM, Hirschhauser C, Waldmann C, Schmalz HG, ten Berg JM, Taubert D: Paraoxonase- 1 is a major determinant of clopidogrel efficacy. Nat Med 2011, 17:110-116.

35. Sibbing D, Koch W, Massberg S, Byrne RA, Mehilli J, Schulz S, Mayer K, Bernlochner I, Schomig A, Kastrati A: No association of paraoxonase-1 Q192R genotypes with platelet response to clopidogrel and risk of stent thrombosis after coronary stenting. Eur Heart J 2011, 32:1605-1613.

36. Bhatt $\mathrm{DL}$ : Is prasugrel superior to clopidogrel for patients with acute coronary syndromes undergoing PCI? Nat Clin Pract Cardiovasc Med 2008, 5:252-253.

37. Von-Lewinski F, Riggert J, Paulus W: Prasugrel versus clopidogrel. N Engl J Med 2008, 358:1299. author reply 1299-1301.

38. Pasceri V, Patti G, Di Sciascio G: Prasugrel versus clopidogrel. N Engl J Med 2008, 358:1298-1299. author reply 1299-1301.

39. Serebruany V: Prasugrel versus clopidogrel. N Engl J Med 2008, 358:1298. author reply 1299-1301.

40. Wiviott SD, Trenk D, Frelinger AL, O'Donoghue M, Neumann FJ, Michelson AD, Angiolillo DJ, Hod H, Montalescot G, Miller DL, et al: Prasugrel compared with high loading- and maintenance-dose clopidogrel in patients with planned percutaneous coronary intervention: the prasugrel in comparison to clopidogrel for inhibition of platelet activation and aggregation-thrombolysis in myocardial infarction 44 trial. Circulation 2007, 116:2923-2932.

41. Wiviott SD, Braunwald E, McCabe CH, Montalescot G, Ruzyllo W, Gottlieb S, Neumann FJ, Ardissino D, De Servi S, Murphy SA, et al: Prasugrel versus clopidogrel in patients with acute coronary syndromes. N Engl J Med 2007, 357:2001-2015.

42. Scott SA, Sangkuhl K, Gardner EE, Stein CM, Hulot JS, Johnson JA, Roden DM, Klein TE, Shuldiner AR: Clinical pharmacogenetics implementation consortium guidelines for cytochrome P450-2C19 (CYP2C19) genotype and clopidogrel therapy. Clin Pharmacol Ther 2011, 90:328-332

43. Reese ES, Daniel Mullins C, Beitelshees AL, Onukwugha E: Costeffectiveness of cytochrome P450 2C19 genotype screening for selection of antiplatelet therapy with clopidogrel or prasugrel. Pharmacotherapy 2012, 32:323-332.

44. Guzauskas GF, Hughes DA, Bradley SM, Veenstra DL: A risk-benefit assessment of prasugrel, clopidogrel, and genotype-guided therapy in patients undergoing percutaneous coronary intervention. Clin Pharmacol Ther 2012, 91:829-837.

45. Cannegieter SC, Rosendaal FR, Wintzen AR, van der Meer FJ, Vandenbroucke JP, Briet E: Optimal oral anticoagulant therapy in patients with mechanical heart valves. N Engl I Med 1995, 333:11-17.

46. Obayashi K, Nakamura K, Kawana J, Ogata H, Hanada K, Kurabayashi M, Hasegawa A, Yamamoto K, Horiuchi R: VKORC1 gene variations are the major contributors of variation in warfarin dose in Japanese patients. Clin Pharmacol Ther 2006, 80:169-178.

47. Tellers DM, McWilliams JC, Humphrey G, Journet M, DiMichele L, Hinksmon J, McKeown AE, Rosner T, Sun Y, Tillyer RD: On the mechanism of an asymmetric alpha, beta-unsaturated carboxylic acid hydrogenation: application to the synthesis of a PGD2 receptor antagonist. J Am Chem Soc 2006, 128:17063-17073.

48. Sanderson S, Emery J, Higgins J: CYP2C9 gene variants, drug dose, and bleeding risk in warfarin-treated patients: a HuGEnet systematic review and meta-analysis. Genet Med 2005, 7:97-104.

49. D'Andrea G, D'Ambrosio RL, Di Perna P, Chetta M, Santacroce R, Brancaccio V, Grandone E, Margaglione M: A polymorphism in the VKORC1 gene is associated with an interindividual variability in the dose-anticoagulant effect of warfarin. Blood 2005, 105:645-649.

50. Wadelius M: Point: use of pharmacogenetics in guiding treatment with warfarin. Clin Chem 2009, 55:709-711.

51. Saminathan R, Bai J, Sadrolodabaee L, Karthik GM, Singh O, Subramaniyan K, Ching CB, Chen WN, Chowbay B: VKORC1 pharmacogenetics and 
pharmacoproteomics in patients on warfarin anticoagulant therapy: transthyretin precursor as a potential biomarker. PLoS One 2010, 5:e15064.

52. Johnson JA, Gong L, Whirl-Carrillo M, Gage BF, Scott SA, Stein CM, Anderson JL, Kimmel SE, Lee MT, Pirmohamed M, et al: Clinical pharmacogenetics implementation consortium guidelines for CYP2C9 and VKORC1 genotypes and warfarin dosing. Clin Pharmacol Ther 2011, 90:625-629.

53. Lenzini PA, Grice GR, Milligan PE, Dowd MB, Subherwal S, Deych E, Eby CS, King CR, Porche-Sorbet RM, Murphy CV, et al: Laboratory and clinical outcomes of pharmacogenetic vs. clinical protocols for warfarin initiation in orthopedic patients. J Thromb Haemost 2008, 6:1655-1662.

54. Caraco Y, Blotnick S, Muszkat M: CYP2C9 genotype-guided warfarin prescribing enhances the efficacy and safety of anticoagulation: a prospective randomized controlled study. Clin Pharmacol Ther 2008, 83:460-470.

55. Ezekowitz MD, Connolly S, Parekh A, Reilly PA, Varrone J, Wang S, Oldgren J, Themeles E, Wallentin L, Yusuf S: Rationale and design of RE-LY: randomized evaluation of long-term anticoagulant therapy, warfarin, compared with dabigatran. Am Heart J 2009, 157:805-810. 810 e801-802

56. Freeman JV, Zhu RP, Owens DK, Garber AM, Hutton DW, Go AS, Wang PJ, Turakhia MP: Cost-effectiveness of dabigatran compared with warfarin for stroke prevention in atrial fibrillation. Ann Intern Med 2011, 154:1-11.

57. Kamel H, Johnston SC, Easton JD, Kim AS: Cost-effectiveness of dabigatran compared with warfarin for stroke prevention in patients with atrial fibrillation and prior stroke or transient ischemic attack. Stroke 2012, 43:881-883.

58. Sorensen SV, Kansal AR, Connolly S, Peng S, Linnehan J, Bradley-Kennedy C, Plumb JM: Cost-effectiveness of dabigatran etexilate for the prevention of stroke and systemic embolism in atrial fibrillation: a Canadian payer perspective. Thromb Haemost 2011, 105:908-919.

59. Lutter R, McWilliams A, Nardinelli C: Health care savings from personalized medicine using genetic testing: the case of warfarin. In Book health care savings from personalized medicine using genetic testing: the case of warfarin; 2006.

60. Sica DA: ACE inhibitors and stroke: new considerations. J Clin Hypertens (Greenwich) 2002, 4:126-129. 133.

61. Brunham LR, Lansberg PJ, Zhang L, Miao F, Carter C, Hovingh GK, Visscher H, Jukema JW, Stalenhoef AF, Ross CJ, et al: Differential effect of the rs4149056 variant in SLCO1B1 on myopathy associated with simvastatin and atorvastatin. Pharmacogenomics J 2011, 12:233-237.

62. Link E, Parish S, Armitage J, Bowman L, Heath S, Matsuda F, Gut I, Lathrop M, Collins R: SLCO1B1 variants and statin-induced myopathy-a genomewide study. N Engl J Med 2008, 359:789-799.

63. Wilke RA, Ramsey LB, Johnson SG, Maxwell WD, McLeod HL, Voora D, Krauss RM, Roden DM, Feng Q, Cooper-Dehoff RM, et al: The clinical pharmacogenomics implementation consortium: CPIC guideline for SLCO1B1 and simvastatin-induced myopathy. Clin Pharmacol Ther 2012, 92:112-117

64. Medication use among senior Canadians. http://www.statcan.gc.ca/pub/82003-x/2009001/article/10801-eng.pdf.

65. Johansson I, Ingelman-Sundberg M: Genetic polymorphism and toxicologywith emphasis on cytochrome p450. Toxicol Sci 2011, 120:1-13.

66. Carlson TJ, Fisher MB: Recent advances in high throughput screening for ADME properties. Comb Chem High Throughput Screen 2008, 11:258-264.

\section{Submit your manuscript to a SpringerOpen ${ }^{\circ}$ journal and benefit from:}

- Convenient online submission

- Rigorous peer review

- Immediate publication on acceptance

- Open access: articles freely available online

- High visibility within the field

- Retaining the copyright to your article 